Journal of Algebra and Its Applications

(2019) 1950188 (13 pages)

(C) World Scientific Publishing Company

DOI: $10.1142 / \mathrm{S} 0219498819501883$

\title{
On the structure of modules defined by subinjectivity
}

\author{
Ferhat Altinay*,‡, Engin Büyükaşık*, ${ }^{*}$ and Yılmaz Durg̃un ${ }^{\dagger}, \boldsymbol{\uparrow}$ \\ *Department of Mathematics, Izmir Institute of Technology \\ Gülbahçeköyü 35430, Urla, Izmir, Turkey \\ ${ }^{\dagger}$ Department of Mathematics, Çukurova University \\ 01330, Adana, Turkey \\ ¥ferhataltinay@iyte.edu.tr \\ §enginbuyukasik@iyte.edu.tr \\ 『ydurgun@cu.edu.tr
}

Received 25 May 2018

Accepted 21 September 2018

Published 2 November 2018

Communicated by S. R. López-Permouth

\begin{abstract}
The aim of this paper is to present new results and generalize some results about indigent modules. The commutative rings whose simple modules are indigent or injective are fully determined. The rings whose cyclic right modules are indigent are shown to be semisimple Artinian. We give a complete characterization of indigent modules over commutative hereditary Noetherian rings. We show that a reduced module is indigent if and only if it is a Whitehead test module for injectivity over commutative hereditary noetherian rings. Furthermore, Dedekind domains are characterized by test modules for injectivity by subinjectivity.
\end{abstract}

Keywords: Injective modules; subinjectivity domain; indigent modules.

Mathematics Subject Classification: 16D10, 16D40, 16D60, 16E99

\section{Introduction}

Throughout, $R$ will denote an associative ring with identity, and modules will be unital right $R$-modules, unless otherwise stated. As usual, we denote by Mod $-R$ the category of right $R$-modules. A module $M$ is said to be $N$-injective if for every submodule $K \leq N$ and every morphism $f: K \rightarrow M$ there exists a morphism $\bar{f}: N \rightarrow M$ such that $\left.\bar{f}\right|_{K}=f$. For a module $M$, the injectivity domain of $M$, $\mathfrak{I n}^{-1}(M)$, is defined to be the collection of all modules $N$ such that $M$ is $N$-injective, that is $\mathfrak{I n}^{-1}(M)=\{N \in \operatorname{Mod}-R \mid M$ is $N$-injective $\}$. It is evident that every module is injective relative to semisimple modules. Thus, for any $R$-module $M$, the injectivity domain $\mathfrak{I n}^{-1}(M)=\{N \in \operatorname{Mod}-R \mid M$ is $N$-injective $\}$ of $M$ contains all

$\S$ Corresponding author. 
semisimple right $R$-modules. For more information about the injectivity domains, we refer the reader to [6]. In [1], Alahmadi et al. initiated the study of poor modules, namely modules whose injectivity domains consist only of semisimple modules in Mod $-R$.

The study of poor modules and rings they serve to characterize has experienced growing interest in recent years (see [8, 11, 12, 19]). Searching for an alternative notion which is also an opposite of injectivity, the notions of subinjectivity and of subinjectivity domain were introduced in [8] and they served to introduce the so-called indigent modules. Given two right modules $M$ and $N, M$ is said to be $N$-subinjective if for every extension $K$ of $N$ and every homomorphism $f: N \rightarrow M$ there exists a homomorphism $h: K \rightarrow M$ such that $\left.h\right|_{N}=f$. The subinjectivity domain of $M, \underline{\mathfrak{I n}}^{-1}(M)$, is defined to be the collection of all modules $N$ such that $M$ is $N$-subinjective, that is $\underline{\mathfrak{I n}}^{-1}(M)=\{N \in \operatorname{Mod}-R \mid M$ is $N$-subinjective $\}$. It is clear that a module $M$ is injective if and only if $\mathfrak{I n n}^{-1}(M)=\operatorname{Mod}-R$. If $N$ is injective, then $M$ is vacuously $N$-subinjective. So, the smallest possible subinjectivity domain is the class of injective modules. A module with such a subinjectivity domain is defined in [7] to be indigent. In recent years, there is a considerable interest in indigent modules and rings defined via these modules (see [4, 7, 9, 10, 18]).

In [27, a module $M$ is said to be a Whitehead test module for injectivity (or i-test) if $N$ is injective whenever $\operatorname{Ext}_{R}^{1}(M, N)=0$. In that paper, a non-semisimple ring $R$ is said to be fully saturated provided that all non-projective modules are i-test. In [4, Theorem 16], the authors proved that if a ring $R$ is not von Neumann regular, then $R$ is right fully saturated if and only if all non-injective modules are indigent.

The aim of this paper is to present new results and to generalize some results about indigent modules. In Sec. 2, we prove that ring is right hereditary if and only if the subinjectivity domain of any right module is closed under factor modules (Theorem 2.1). Recall that, a ring $R$ is right small if it is a small submodule of its injective hull. We prove that over a right small ring, the direct sum of a complete set of representatives of simple modules is indigent if and only if every module without maximal submodules is injective, in which case the (underlying) ring is necessarily right hereditary and right Noetherian. We prove that over a right small ring every simple right $R$-module is indigent or injective if and only if one of the following cases hold: (i) $R$ is a right $\mathrm{V}$-ring, (ii) for any right $R$-module $M, M$ is indigent or, $\operatorname{Soc}(M)=\operatorname{Soc}(N)$, where $N$ is a maximal injective submodule of $M$. A right module is called reduced if it has no nonzero injective submodule. Over a commutative hereditary Noetherian ring, a module $M$ is indigent if and only if, the singular submodule $Z(M)$ of $M$ is indigent if and only if the reduced part $M^{\prime}$ of $M$ contains a copy of each singular simple module. Consequently, a reduced module is indigent if and only if, it is an i-test module. In [7, Proposition 4.13], it is shown that if every nonzero cyclic right $R$-module is indigent, then $R$ is right semiartinian. We prove that the rings whose cyclic right modules are indigent are semisimple Artinian. 
In Sec. 3] we characterize commutative rings over which each simple module is indigent or injective. Namely we prove that, over a commutative ring $R$, every simple module is indigent or injective if and only if $R$ is a $V$-ring, or $R=A \times B$, where $B$ is semisimple, and $A$ is the zero ring, a DVR, or a local $Q F$-ring.

A right module $N$ is said to be a test module for injectivity by subinjectivity (t.i.b.s., for short) if whenever $M$ is $N$-subinjective for some right module $M$, then $M$ is injective (see [4]). In the last section, we give a characterization of t.i.b.s. modules over Dedekind domains. We prove that, a commutative domain $R$ is Dedekind if and only if every nonzero ideal of $R$ is a t.i.b.s. module if and only if a nonzero $R$-module $M$ is t.i.b.s. exactly when $\operatorname{Hom}(M, R) \neq 0$.

For a module $M, \operatorname{Soc}(M)$, and $\operatorname{Rad}(M)$ denote the socle and the Jacobson radical of $M$, respectively. $Z(M)$ will denote the singular submodule of $M$. For any ring in our discussion, $J$ will stand for the Jacobson radical of that ring. For a module $M$, the character module $\operatorname{Hom}_{\mathbb{Z}}(M, \mathbb{Q} / \mathbb{Z})$ is denoted by $M^{+}$, and the injective hull is denoted by $E(M)$. For all other basic or background material, we refer the reader to [6] 17.

\section{Indigent Modules Over Hereditary Noetherian Rings}

In this section, we give a characterization of indigent modules over commutative hereditary Noetherian rings. We prove that a right small ring is right hereditary right Noetherian if the direct sum of simple right modules is indigent. We prove that over a right small ring every simple right $R$-module is indigent or injective if and only if $R$ is a right $\mathrm{V}$-ring or, for any right $R$-module $M, M$ is indigent or $\operatorname{Soc}(M)=\operatorname{Soc}(N)$, where $N$ is the largest injective submodule of $M$. We prove that the rings whose cyclic right modules are indigent are semisimple Artinian.

We begin with the following result which is crucial for proving the structure of indigent modules over commutative hereditary Noetherian rings. The injectivity domain of any right module is closed under homomorphic images over any ring. The following result shows that corresponding result for the subinjectivity domains holds only over right hereditary rings. Note also that the following result generalize [7] Proposition 2.5(3)].

Theorem 2.1. A ring $R$ is right hereditary if and only if the subinjectivity domain of each right module is closed under homomorphic images.

Proof. Suppose $M$ is $N$-subinjective for right modules $M$ and $N$. Let $K \leq N$ and $f: N / K \rightarrow M$ be a homomorphism. Consider the canonical epimorphisms $\pi: N \rightarrow N / K$ and $\pi^{\prime}: E(N) \rightarrow E(N) / K$. Since $M$ is $N$-subinjective, there is a $\varphi$ : $E(N) \rightarrow M$ such that $\varphi_{\left.\right|_{N}}=f \pi$. Clearly $f \pi(K)=0$, and so $K \leq \operatorname{Ker}(\varphi)$. Then, by factor theorem, there exist a $\varphi^{\prime}: E(N) / K \rightarrow M$, given by $\varphi^{\prime}(a+K)=\varphi(a)$ for each $a \in E(N)$. Clearly, $\varphi_{\left.\right|_{N / K} ^{\prime}}^{\prime}=f$. Then $M$ is $N / K$-subinjective by [7, Lemma 2.2(4)]. This proves the necessity. Conversely suppose the subinjectivity domain of every right module is closed under homomorphic images. Let $E$ be an injective module 
and $K \leq E$. Clearly $E / K$ is $E$-subinjective. Therefore $E / K$ is $E / K$-subinjective by the assumption. Hence $E / K$ is injective. Thus $R$ is right hereditary.

If $\mathcal{C}$ is a class of modules, then $\mathbf{T}_{\mathcal{C}}=\left(\mathcal{T}_{\mathcal{C}}, \mathcal{F}_{\mathcal{C}}\right)$, where $\mathcal{T}_{\mathcal{C}}=\{M \mid \operatorname{Hom}(M, C)=0$ for every $C \in \mathcal{C}\}$ is said to be the torsion theory cogenerated by $\mathcal{C}$.

We have the following for $\mathcal{C}=\{M\}$, which can be verified easily.

Lemma 2.2. Let $R$ be a ring and $M$ be a right $R$-module. Assume that every injective right $R$-module is in $\mathcal{T}_{M}$. Then $M$ is $N$-subinjective if and only if $\operatorname{Hom}(N, M)=0$.

Corollary 2.3. Let $R$ be a right hereditary ring and $M$ be a reduced right $R$-module. Then $M$ is $N$-subinjective if and only if $\operatorname{Hom}(N, M)=0$.

Proposition 2.4. Let $R$ be a ring and $M$ be a right $R$-module. Assume that every injective right $R$-module is in $\mathcal{T}_{M}$. The following are equivalent.

(1) $M$ is indigent.

(2) $\mathcal{T}_{M}=\{N \mid N$ is injective right $R$-module $\}$.

In particular, $R$ is right hereditary, right Noetherian.

A ring $R$ is said to be right small if $R_{R}$ is a small submodule in its injective hull $E(R)$, i.e. $R_{R} \ll E\left(R_{R}\right)$. Every commutative domain is a small ring (see [24] Folgerung 5.3]). We recall the following characterization of small rings.

Proposition 2.5 ([26, 3.3: 24, 4.8]). Let $R$ be a ring and let $E(R)$ be the injective hull of $R_{R}$. Then the following conditions are equivalent.

(1) $R$ is a right small ring.

(2) $\operatorname{Rad}(E)=E$ for every injective right $R$-module $E$.

(3) $\operatorname{Rad}(E(R))=E(R)$.

Let $\mathcal{C}$ be a complete set of nonisomorphic simple right $R$-modules. Then $\mathcal{T}_{\mathcal{C}}=$ $\{M \mid \operatorname{Rad}(M)=M\}$. Over the ring of integers $\bigoplus \mathbb{Z}_{p}$, where $p$ ranges over the prime integers, is indigent (see [7]). Over right small ring we have the following by Lemma 2.2. Propositions 2.4 and 2.5

Corollary 2.6. Let $R$ be a right small ring and $\mathcal{C}$ be a complete set of nonisomorphic simple right $R$-modules. Set $S=\bigoplus_{U \in \mathcal{C}} U$. The following are equivalent.

(1) $S$ is indigent.

(2) Any module with $\operatorname{Rad}(N)=N$ is injective.

In particular, $R$ is right hereditary, right Noetherian.

Proposition 2.7. Let $R$ be a right small ring. The following are equivalent.

(1) Every simple right $R$-module is indigent or injective. 
(2) $R$ is a right $V$-ring or, for any right $R$-module $M, M$ is indigent or $M$ has a maximal injective submodule and $\operatorname{Soc}(M)=\operatorname{Soc}(N)$, where $N$ is a maximal injective submodule of $M$.

Proof. $(2) \Rightarrow(1)$ is clear. (1) $\Rightarrow(2)$ Suppose every simple right $R$-module is indigent or injective. If all simple modules are injective, then $R$ is a $V$-ring. Now suppose, there is a non-injective simple right $R$-module $U$. Then $U$ is indigent by the hypothesis. If $U^{\prime}$ is any simple module which is not isomorphic to $U$, then $\operatorname{Hom}\left(U^{\prime}, U\right)=0$. That is, $U$ is $U^{\prime}$-subinjective, and so $U^{\prime}$ must be injective because $U$ is indigent. Thus the ring has a unique non-injective simple module up to isomorphism, say $U$. By Corollary 2.6 $R$ is right hereditary, right Noetherian. Let $M$ be a right $R$-module. Then $M=N \oplus M^{\prime}$ for some $N, M^{\prime} \leq M$, where $M^{\prime}$ is the reduced part of $M$ and $N$ is a maximal injective submodule of $M$. Note that $\operatorname{Hom}\left(U^{\prime}, M^{\prime}\right)=0$ for every injective simple right $R$-module $U^{\prime}$. Now, if $\operatorname{Hom}\left(U, M^{\prime}\right)=0$ then $\operatorname{Soc}\left(M^{\prime}\right)=0$, and hence $\operatorname{Soc}(M)=\operatorname{Soc}(N)$. Else $M^{\prime}$ contains a copy of $U$. In this case let us see that $M^{\prime}$ is indigent. Assume the contrary that $M^{\prime}$ is not indigent. Then $M^{\prime}$ is $K$-subinjective for some non-injective right module $K$. As $U$ is indigent, $\operatorname{Hom}(K, U) \neq 0$. Then $M^{\prime}$ is $U$-subinjective by Theorem 2.1. So $\operatorname{Hom}\left(U, M^{\prime}\right)=0$ by Corollary 2.3 a contradiction. Therefore, $M^{\prime}$ is indigent, and so is $M$.

Proposition 2.8. Let $R$ be a commutative ring and $S$ be a semisimple module. Consider the following statements.

(1) $S$ is injective.

(2) $S$ is flat.

(3) $S$ is projective.

Then $(1) \Rightarrow(2) \Leftarrow(3)$. If $R$ is also Noetherian, then $(1) \Leftrightarrow(2) \Leftrightarrow(3)$.

Proof. $(1) \Rightarrow(2)$ By (28), Lemma 2.6) and by the fact that direct sum of flat modules is flat. (3) $\Rightarrow(2)$ Projective modules are flat, so this is clear. (2) $\Rightarrow$ (1) Over a Noetherian ring an arbitrary direct sum of injective modules is injective. So the proof is clear by ([28, Lemma 2.6]). (2) $\Rightarrow(3)$ If $R$ is Noetherian, then each simple module is finitely presented. Finitely presented flat modules are projective by [17, Theorem 4.30]. Since direct sum of projective modules is projective, semisimple flat modules are projective over Noetherian rings.

Theorem 2.9 ([21, Theorem 4.6]). A hereditary Noetherian ring $R$ is a finite direct sum of Artinian hereditary rings and hereditary Noetherian prime rings.

Proposition 2.10. Let $R$ be a commutative hereditary Noetherian ring. Then every module with $\operatorname{Rad}(N)=N$ is injective.

Proof. By Theorem 2.9 and the commutativity assumption, we have $R=e_{1} R \oplus$ $\cdots e_{t} R \oplus f_{1} R \oplus \cdots f_{k} R$, where $e_{i} R$ are fields and $f_{j} R$ are Dedekind domains for 
$1 \leq i \leq t$ and $1 \leq j \leq k$. Let $S=\bigoplus_{i=1}^{t} e_{i} R$ and $T=\bigoplus_{j=1}^{k} f_{j} R$. Let $E$ be a module with $\operatorname{Rad}(E)=E$. Then $E$ can not have a simple direct summand, otherwise we would have $\operatorname{Rad}(E) \neq E$. Thus $S . E=0$, and so $E$ is a $T$-module. So, $E$ has a decomposition as $E=f_{1} E \oplus f_{2} E \oplus \cdots \oplus f_{k} E$, where $f_{j} E$ is an $f_{j} R$ module and $\operatorname{Rad}\left(f_{i} R\right)=f_{i} R$ for $j=1, \ldots, k$. Since $f_{j} R$ is a Dedekind domain and $\operatorname{Rad}\left(f_{j} E\right)=f_{j} E$, the modules $f_{j} E$ are injective $f_{j} R$-modules for each $j=1, \ldots, k$ (see, [2 Lemma 4.4]). Thus $E$ is injective both as a $T$-module and as $R$-module (see, [17, Example 3.11A]).

Another consequence of Theorem 2.9, is the following proposition.

Proposition 2.11. Let $R$ be a commutative hereditary Noetherian ring. Then $R=$ $S \oplus T$, where $S$ is semisimple, $\operatorname{Soc}(T)=0$ and $T$ is a right small ring.

Proof. By Theorem 2.9, $R=S \oplus T$, where $S=\operatorname{Soc}(R)$ and $T$ is a direct sum of Dedekind domains. Now let us prove that $T$ is a right small ring. Assume w.l.o.g. that $T \neq 0$. If $T$ is not a small ring, then as $T$ is cyclic $T \nsubseteq \operatorname{Rad}(E(T))$. So $T+K=E(T)$ for some maximal submodule $K$ of $E(T)$. Then $T /(T \cap K) \cong$ $E(T) / K$ is simple, and also injective by the hereditary condition. Thus $T /(T \cap K)$ is projective by Proposition [2.8, and so $T=T \cap K \oplus U$ for some simple submodule $U$ of $R$. Then $U \leq \operatorname{Soc}(T)=0$, a contradiction. Therefore $T$ is a small submodule of $E(T)$, i.e. $T$ is a right small ring.

Proposition 2.12. Let $R$ be a commutative hereditary Noetherian ring and $C$ be the direct sum of nonisomorphic singular simple $R$-modules. Then $C$ is indigent.

Proof. Let $R=S \oplus T$ be as in Proposition 2.11. Since $S$ is projective it is nonsingular. Thus we have $S C=0$, and so $C$ is a $T$-module. Clearly every simple $T$-module is singular. Therefore $C$ is exactly the direct sum nonisomorphic singular simple $T$-modules. Now $T$ is a small ring. Thus $C$ is an indigent $T$-module by Corollary 2.6 and Proposition 2.10 Now let us see that $C$ is an indigent $R$-module. Suppose $C$ is $N$-subinjective for some $R$-module $N$. There is a decomposition $N=N \cdot S \oplus N \cdot T$. Since $S$ is projective it is injective by 2.8. Then $N \cdot S$ is injective. Since $C$ is $N \cdot T$ subinjective and $C$ is indigent $T$-module, $N \cdot T$ is injective $T$-module. Now it is straightforward to check that both $N \cdot S$ and $N \cdot T$ are injective as $R$-modules. Hence their direct sum $N=N \cdot S \oplus N \cdot T$ is injective $R$-module (see [17, Exercise $3.11 \mathrm{~A}]$ ). Therefore $C$ is an indigent $R$-module.

Proposition 2.13. Let $R$ be a commutative Noetherian ring and $M$ be an indigent $R$-module. Let $C$ be the direct sum of nonisomorphic singular simple $R$-modules. Then $M$ contains a submodule isomorphic to $C$.

Proof. Note that a singular simple module is non-injective by Proposition 2.8 Suppose $\operatorname{Hom}(U, M)=0$ for some singular simple module $U$. This implies that $M$ is 
$U$-subinjective. This contradicts the fact that $M$ is indigent. Thus $\operatorname{Hom}(U, M) \neq 0$ for each singular simple module $U$. This implies that $M$ contains a submodule isomorphic to $C$.

Proposition 2.14. Let $R$ be a commutative hereditary Noetherian ring and $M$ an $R$-module and $M^{\prime}$ be its reduced part. The following are equivalent.

(1) $M$ is indigent.

(2) $M^{\prime}$ is indigent.

(3) $M^{\prime}$ contains a copy of each simple singular module.

(4) $Z\left(M^{\prime}\right)$ is indigent.

(5) $Z(M)$ is indigent.

Proof. $(1) \Leftrightarrow(2)$ Since $R$ is Noetherian, the module $M$ has a maximal injective submodule, say $N$. Then $M=M^{\prime} \oplus N$, for some $M^{\prime} \leq M$. Now it is easy to see that, for a right module $K, M$ is $K$-subinjective if and only if $M^{\prime}$ is $K$-subinjective. Thus $M$ is indigent if and only if $M^{\prime}$ is indigent. (2) $\Rightarrow(3)$ Follows by Proposition [2.13, $(3) \Rightarrow(2)$ Suppose $M^{\prime}$ is $K$-subinjective for some $R$-module $K$. Assume that $K$ is not injective. Then w.l.o.g. we can assume that $K$ has no nonzero injective submodule by the Noetherian condition. Note that $\operatorname{Rad}(K) \neq K$ by Proposition 2.10. By Proposition 2.8 and our assumption $K$ essentially contains a maximal submodule $L$. Since $M^{\prime}$ is $K$-subinjective, $M^{\prime}$ is $K / L$-subinjective by Theorem 2.1. Moreover, by (3) we have $\operatorname{Hom}\left(K / L, M^{\prime}\right) \neq 0$. Thus there is a nonzero $g: E(K / L) \rightarrow M^{\prime}$. Then $g(E(K / L))$ is a nonzero injective submodule of $M^{\prime}$. Contradiction, because $M^{\prime}$ is reduced. Therefore $K$ must be injective and so $M^{\prime}$ is indigent. (3) $\Rightarrow$ (4) Similar to the proof of $(3) \Rightarrow(2)$. (4) $\Rightarrow(3)$ By Proposition 2.13. (4) $\Leftrightarrow$ (5) Since $M=M^{\prime} \oplus N, Z(M)=Z\left(M^{\prime}\right) \oplus Z(N)$. Since $Z(N)$ is closed submodule of the injective module $N$, it is also injective. Therefore, the subinjectivity domains of $Z(M)$ and $Z\left(M^{\prime}\right)$ are the same. Hence, $Z(M)$ is indigent if and only if $Z\left(M^{\prime}\right)$ is indigent.

In [27], Trlifaj defined what he called a Whitehead test module for injectivity (i-test module) as follows: A module $M$ is an i-test module if $N$ is injective whenever $\operatorname{Ext}_{R}^{1}(M, N)=0$. A ring $R$ is called right $V$-ring if each simple right $R$-module is injective. A ring $R$ is called a right $G V$-ring if every simple right module is either injective or projective.

Proposition 2.15. Let $R$ be a two-sided hereditary Noetherian ring which is not $G V$-ring. A reduced indigent right $R$-module is an $i$-test.

Proof. Let $M$ be a reduced indigent right $R$-module. Then $\operatorname{Hom}(S, M) \neq 0$ for every singular simple right $R$-module $S$. Then, $M$ is i-test by [5, Theorem 4.19]. 
The following result follows by Propositions 2.14, and 2.15] and [5, Theorem 4.19].

Corollary 2.16. Let $R$ be a commutative hereditary Noetherian ring. An $R$-module $M$ is indigent if and only if the reduced part $M^{\prime}$ of $M$ is indigent if and only if the reduced part $M^{\prime}$ of $M$ is an i-test.

In [7. Proposition 4.13], it is shown that if every nonzero cyclic right $R$-module is indigent, then $R$ is right semiartinian. We have the following result.

Proposition 2.17. Let $R$ be ring. Every nonzero cyclic right $R$-module is indigent if and only if $R$ is semisimple artinian.

Proof. Sufficiency is clear. To prove the necessity suppose every nonzero cyclic right $R$-module is indigent. Let $A$ and $B$ be two simple right $R$-modules. Assume that $A$ and $B$ are not isomorphic. Then, $A$ is clearly $B$-subinjective. As $A$ must be indigent by the hypothesis, $B$ is then injective. But, by our assumption, $B$ is also indigent. Then $R$ is semisimple artinian since $B$ is both an injective and indigent module. So, assume w.l.o.g, $R$ has a unique simple module $A$ up to isomorphism. If $A$ is projective, then $R$ is semisimple artinian. Hence, we may assume w.l.o.g. that $A$ is singular. Note that $R$ is also indigent by our assumption. Hence $\operatorname{Hom}(A, R) \neq 0$, otherwise $A$ is injective, a contradiction. Then $R$ has a minimal right ideal, which is isomorphic to $A$, i.e. $R$ is a right Kasch ring. We have the following two cases:

Case I: $\operatorname{Hom}(E(R), A)=0$. Then $R$ is right small ring by Proposition 2.5. By Corollary 2.6, $R$ is right hereditary, contradicting the singularity of $A$.

Case II: $\operatorname{Hom}(E(R), A) \neq 0$. In this case we will show that $R$ is a right self-injective ring: Consider the following diagram

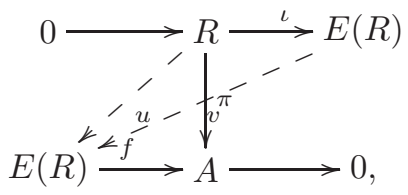

where $\pi$ is an arbitrary (and w.l.o.g. nonzero) map and $f$ is a fixed epimorphism. By projectivity of $R$, there exists a homomorphism $u: R \rightarrow E(R)$ such that $f u=\pi$. Now, by injectivity of $E(R)$, there exists a homomorphism $v: E(R) \rightarrow E(R)$ such that $v \iota=u$. Then $f v \iota=f u=\pi$, and so $A$ is $R$-subinjective. But $A$ is indigent, so $R$ is right self-injective. On the other hand, $R$ is also indigent by the hypothesis. Therefore $R$ is semisimple artinian.

Proposition 2.18. Let $R$ be ring. If every nonzero proper cyclic right $R$-module is indigent, then $R$ is semisimple artinian or $R$ is a local QF ring.

Proof. As in the proof of the previous proposition, $R$ is semisimple artinian or $R$ has a unique simple module which is singular and noninjective. We have the following two cases: 
Case I: $\operatorname{Hom}(E(R), A)=0$. Then $R$ is right small ring by Proposition2.5, We have either $\operatorname{Hom}(A, R) \neq 0$ or $\operatorname{Hom}(A, R)=0$. In the former case, by Proposition 2.6, $R$ is right hereditary ring, and so the simple module $A$ is nonsingular, contradicting the singularity of $A$. In the latter one, $\operatorname{Hom}(A, I)=0$ for every nonzero cyclic right ideal of $R$. Then, by our assumption, $A$ is injective, and so $R$ is semisimple artinian.

Case II: $\operatorname{Hom}(E(R), A) \neq 0$. Following the proof of Proposition 2.17 one can show that $A$ is $P$-subinjective for each projective right $R$-module $P$. But $A$ is indigent, and so $R$ is a QF-ring.

\section{When Simple Modules are Indigent or Injective}

In this section, we give a characterization of the commutative rings whose simple modules are indigent or injective. The following Lemma is well known. We include it for completeness.

Lemma 3.1. If $R$ is a commutative ring, then every simple $R$-module is pureinjective.

Proof. Let $C$ be an injective cogenerator. Then

$$
U^{+}=\operatorname{Hom}(U, C)=\operatorname{Hom}(U, E(U)) \cong U .
$$

Since $U^{+}$is pure-injective, $U$ is pure-injective, too.

By Theorem 2.9, a commutative hereditary Noetherian ring is a direct product of fields and hereditary Noetherian prime rings. Every commutative hereditary Noetherian prime ring is a Dedekind domain. Thus a commutative local hereditary Noetherian ring is a field or DVR. Note that a commutative local hereditary Noetherian ring is a DVR (i.e. a local Dedekind domain). A ring $R$ is called right $\mathrm{H}$-ring if every right module is a direct sum of an injective module and a small module. Every $Q F$-ring is a right $H$-ring by [23, Theorem 4.3].

Theorem 3.2. Let $R$ be a commutative ring. The following are equivalent.

(1) Every simple module is indigent or injective.

(2) $R$ is a $V$-ring, or $R=A \times B$, where $B$ is semisimple, and $A$ is the zero ring, a DVR, or a local $Q F$-ring.

Proof. $(1) \Rightarrow(2)$ Suppose every simple module is indigent or injective. If all simple modules are injective, then $R$ is a $V$-ring. Now suppose, there is a non-injective simple module $U$. Then $U$ is indigent by the hypothesis. If $U^{\prime}$ is any simple module which is not isomorphic to $U$, then $\operatorname{Hom}\left(U^{\prime}, U\right)=0$. That is, $U$ is $U^{\prime}$-subinjective, and so $U^{\prime}$ must be injective because $U$ is indigent. Thus the ring has a unique noninjective simple module, up to isomorphism. Let $\left\{E_{i}\right\}_{i \in I}$ be an arbitrary family of injective modules. Then $\bigoplus_{i \in I} E_{i}$ is a pure submodule of $\prod_{i \in I} E_{i}$. By Lemma 3.1 
the simple module $U$ is pure-injective. Thus $U$ is $\bigoplus_{i \in I} E_{i}$-subinjective. As $U$ is indigent, $\bigoplus_{i \in I} E_{i}$ must be injective. So that the ring $R$ is Noetherian. Now let, $B$ be the sum of the injective simple ideals of $R$. Then $B$ is injective, because the ring is Noetherian. Thus, there is a ring decomposition $R=A \oplus B$ and $U B=0$, so that $U$ is the unique (noninjective) simple $A$-module.

If $X$ is a simple $A$-module, then we must have $X \cong U$. Otherwise $X$ would be injective by the hypothesis. Then $X$ must be projective by Proposition 2.8, Which implies that $A=X^{\prime} \oplus Y$ for some $X^{\prime} \cong X$. But then $X^{\prime} \leq A \cap B=0$. Contradiction. Therefore $A$ has a unique simple module, and this simple is isomorphic to $U$. By the commutativity condition, we get that $A$ is a local ring. We have the following two cases: Case I: $A$ is a small ring, i.e. $A \ll E(A)$. $\operatorname{Then} \operatorname{Rad}(E / K)=E / K$ for each injective $A$-module $E$ and $K \leq E$. Thus $\operatorname{Hom}(E / K, U)=0$, and so $U$ is $E / K$-subinjective. Hence $E / K$ is injective. This proves that $R$ is hereditary. Being local, hereditary and Noetherian implies that, $R$ is a DVR. Case II: $A$ is not small i.e. $\operatorname{Rad}(E(A)) \neq E(A)$. Let us prove that $A$ is $Q F$ by showing that $A$ is injective. We know that $A$ is local and non small. Since $A$ is finitely generated, we have $A \nsubseteq \operatorname{Rad}(E(A))$. Thus there is a maximal submodule $K$ of $E(A)$ such that $A+K=E(A)$. Then $A / A \cap K \cong E(A) / K$ is simple, and so $A \cap K$ is the unique maximal submodule of $A$. Moreover,

$$
E(A) / A \cap K=A / A \cap K \oplus K / A \cap K .
$$

Let $f: A \rightarrow U$ be a nonzero homomorphism. Since $U$ is simple $\operatorname{Ker}(f)=A \cap K$ is maximal. Let $\pi^{\prime}: A \rightarrow A / A \cap K$ and $\pi: E(A) \rightarrow A / A \cap K$ be the natural projections, and $\bar{f}: A / A \cap K \rightarrow U$ be the map satisfying $f=\bar{f} \pi^{\prime}$. It is straightforward to check that the map $g=\bar{f} \pi: E(A) \rightarrow U$ extends $f$ i.e. $\left.g\right|_{A}=f$. Thus $U$ is $A$-subinjective, and so $A$ is injective. Being Noetherian and injective, $A$ is $Q F$.

$(2) \Rightarrow(1)$ If $R$ is a $V$-ring, then each simple module is injective. So that (1) holds. Now assume that $A$ is a DVR. By Proposition $2.8 B$ is an injective $R$-module. Let $U$ be a non-injective simple $R$-module. Then $B \cdot U=0$, and so $U$ is an $A$-module. Since $A$ is a local ring, $U$ is the unique simple $A$-module up to isomorphism. Hence $U$ is an indigent $A$-module by Proposition 2.12. It is routine to see that $U$ is an indigent $R$-module. Now assume that $A$ is a local $Q F$-ring. As in the proof of previous part, the ring $R$ has a unique non-injective simple module, say $U$ which is also the unique simple $A$-module, up to isomorphism. Then since $A$ is local and $Q F, U$ is an indigent $A$-module by [4 Proposition 32; 23 , Theorem 4.4.] Then $U$ is an indigent $R$-module. This completes the proof.

If $R$ is a right Kasch ring which has a simple projective indigent module then, $R$ is semisimple Artinian by [7, Theorem 4.22]. We have the following result on commutative Kasch rings.

Corollary 3.3. Let $R$ be a commutative ring. $R$ is Kasch ring which has a simple singular indigent module if and only if $R$ is local $Q F$. 
Corollary 3.4. Let $R$ be commutative ring. The following are equivalent:

(1) Every simple module is indigent.

(2) $R$ is semisimple, or $R$ is a local ring that is a $V$-ring, a DVR or a $Q F$-ring.

\section{T.I.B.S. Modules Over Commutative Rings}

A right module $N$ is said to be a test module for injectivity by subinjectivity (t.i.b.s., for short) if whenever $M$ is $N$-subinjective for some right module $M$, then $M$ is injective (see [4). The following lemma can be easily proved by using the properties of subinjectivity (see 7]). The proof is omitted here.

Lemma 4.1. The following statements are equivalent for a right $R$-module $M$ :

(1) $M$ is t.i.b.s.

(2) $M^{n}$ is t.i.b.s. for some $n \in \mathbb{Z}^{+}$.

(3) $M^{n}$ is t.i.b.s. for all $n \in \mathbb{Z}^{+}$.

(4) $M \oplus N$ is t.i.b.s. for any right module $N$.

The structure of t.i.b.s. $\mathbb{Z}$-modules is completely characterized in [4]. The following theorem is a generalization of [4, Theorem 21].

Theorem 4.2. The following are equivalent for a commutative domain $R$ :

(1) $R$ is Dedekind.

(2) $R$ is t.i.b.s.

(3) Every nonzero ideal of $R$ is t.i.b.s.

(4) A nonzero $R$-module $M$ is t.i.b.s. exactly when $\operatorname{Hom}(M, R) \neq 0$.

Proof. $(1) \Leftrightarrow(2)$ By [4, Corollary 20].

$(2) \Rightarrow(3)$. Let $I$ be nonzero ideal of $R$. Note that $R$ is Dedekind by the equivalence $(1) \Leftrightarrow(2)$. Then $I \oplus I \cong R \oplus(I \cdot I)$ by [25, Lemma 7.6(i)]. Then (3) follows by Lemma 4.1(2) and (4).

$(3) \Rightarrow(2)$ is clear.

$(3) \Rightarrow(4)$ This follows immediately from the equivalence $(1) \Leftrightarrow(2) \Leftrightarrow(3)$ and Lemma 4.1 .

$(4) \Rightarrow(2) \operatorname{Hom}(R, R) \neq 0$. So $R$ is t.i.b.s. by (4). This proves $(2)$.

\section{Acknowledgment}

The authors are grateful to the anonymous referee for the suggestions and comments that has greatly improved presentation of the paper.

\section{References}

[1] A. N. Alahmadi, M. Alkan and S. López-Permouth, Poor modules: The opposite of injectivity, Glasg. Math. J. 52(A) (2010) 7-17. 
[2] R. Alizade, G. Bilhan and P. F. Smith, Modules whose maximal submodules have supplements, Comm. Algebra 29(6) (2001) 2389-2405.

[3] R. Alizade and E. Büyükaşık, Poor and pi-poor Abelian groups, Comm. Algebra 45(1) (2017) 420-427.

[4] R. Alizade, E. Büyükaşı and N. Er, Rings and modules characterized by opposites of injectivity, J. Algebra 409 (2014) 182-198.

[5] R. Alizade and Y. Durğun, Test modules for flatness, Rend. Semin. Mat. Univ. Padova 137 (2017) 75-91.

[6] F. W. Anderson and K. R. Fuller, Rings and Categories of Modules, Graduate Texts in Mathematics, Vol. 13 (Springer-Verlag, New York, 1974), MR0417223.

[7] P. Aydoğdu and S. R. López-Permouth, An alternative perspective on injectivity of modules, J. Algebra 338 (2011) 207-219.

[8] P. Aydoğdu and B. Saraç, On Artinian rings with restricted class of injectivity domains, J. Algebra 377 (2013) 49-65.

[9] E. Büyükaşık, E. Enochs, J. R. García Rozas, G. Kafkas-Demirci, S. López-Permouth and L. Oyonarte, Rugged modules: The opposite of flatness, Comm. Algebra 46(2) (2018) 764-779.

[10] Y. Durğun, An alternative perspective on flatness of modules, J. Algebra Appl. 15(8) (2016) 1650145.

[11] N. Er, S. López-Permouth and N. Sökmez, Rings whose modules have maximal or minimal injectivity domains, J. Algebra 330 (2011) 404-417.

[12] N. Er, S. López-Permouth and N. K. Tung, Rings whose cyclic modules have restricted injectivity domains, J. Algebra 466 (2016) 208-228.

[13] A. Facchini, Module Theory (Birkhauser, 1998).

[14] L. Fuchs, Neat submodules over integral domains, Period. Math. Hungar. 64(2) (2012) 131-143.

[15] L. Fuchs and L. Salce, Modules Over Non-Noetherian Domains (American Mathematical Society, 2001).

[16] K. R. Goodearl, Singular Torsion and the Splitting Properties (American Mathematical Society, 1972).

[17] T. Y Lam, Lectures on Modules and Rings, Graduate Texts in Mathematics, Vol. 189 (Springer-Verlag, New York, 1999).

[18] S. R. López-Permouth, J. Mastromatteo, Y. Tolooei and B. Üngör, Pure-injectivity from a different perspective, Glasg. Math. J. 60(1) (2018) 135-151.

[19] S. R. López-Permouth and J. E. Simental, Characterizing rings in terms of the extent of the injectivity and projectivity of their modules, J. Algebra 362 (2012) 56-69.

[20] E. Matlis, Injective modules over Noetherian rings, Pacific J. Math. 8 (1958) 511-528, MR0099360.

[21] J. C. McConnell and J. C. Robson, Noncommutative Noetherian Rings (American Mathematical Society, Providence, RI, 2000).

[22] S. H. Mohamed and B. J. Müller, Continuous and Discrete Modules, London Mathematical Society Lecture Note Series, Vol. 147 (Cambridge University Press, Cambridge, 1990).

[23] K. Oshiro, Lifting modules, extending modules and their applications to QF-rings, Hokkaido Math. J. 13(3) (1984) 310-338.

[24] B. Pareigis, Radikale und kleine Moduln (Radicals and small modules), Bayer. Akad. Wiss. Math. Natur. Kl. S.-B. 2 (1965) 185-199.

[25] D. Passman, A Course in Ring Theory (American Mathematical Society, Chelsea Publishing, 2004). 
[26] V. S Ramamurthi, The smallest left exact radical containing the Jacobson radical, Ann. Soc. Sci. Bruxelles Sér. I 96 (1982) 201-206.

[27] J. Trlifaj, Whitehead test modules, Trans. Amer. Math. Soc. 348(4) (1996) 15211554.

[28] R. Ware, Endomorphism rings of projective modules, Trans. Amer. Math. Soc. 155 (1971) 233-256. 